Original Research Paper

\title{
Validation of Global Financial Crisis on Bursa Malaysia Stocks Market Companies via Covariance Structure
}

\author{
Shamshuritawati Sharif, Suzilah Ismail, Zurni Omar and Low Huey Theng \\ School of Quantitative Sciences, Universiti Utara Malaysia, Sintok, Malaysia
}

Article history

Received: 18-05-2016

Revised: $25-10-2016$

Accepted: $27-10-2016$

Corresponding Author: Shamshuritawati Sharif School of Quantitative Sciences, Universiti Utara Malaysia, Sintok, Malaysia Email: shamshurita@uum.edu.my

\begin{abstract}
The global financial crisis of 2007 to 2009 affects the economic development around the world. It started with US in 2007 and followed by Malaysia in 2008. The purpose of this study is to validate the impact before and beginning of the crisis on seventy seven Bursa Malaysia stocks market companies. Two data sets of 2007 and 2008 were used in testing the differences of the covariance structures by using a new test known as $S^{*}$ statistic that been developed for high dimensional data set such as the stock market. The test revealed that the covariance structure of 2007 and 2008 significantly different from each other. Thus, Optimal Minimum Spanning Tree (OMST), degree centrality measure and network topology were implemented in identifying the companies that contribute to the different covariance structure. The finding shows that HWAN is the most dominant for 2007 and MRES for 2008. The rise or fall (instability) of HWAN and MRES gave large impact on the stability structure of the stock market. The global financial crisis in 2008 affected HWAN but HONG seems to maintain in the network.
\end{abstract}

Keywords: Covariance Test, Degree Centrality, High Dimension, Optimal Minimum Spanning Tree, Stock Market

\section{Introduction}

The global financial crisis of 2007 to 2009 which started in 2007 with US as the epicentre is the worst financial crisis since the Great Recession in 1930s (Ergungor and Cherny, 2009). Economies all around the world experienced a sharp fall in the Gross Domestic Product (GDP). In emerging and developing countries, real GDP growth fell from $8.3 \%$ in 2007 to $6.1 \%$ in 2008 and just $2.4 \%$ in 2009 . The GDP of 2009 marked as the most serious shortfalls in all countries (Dolphin and Chappell, 2010).

Malaysia was also affected where the GDP dropped from $7.6 \%$ in the first quarter of 2008 to $-6.2 \%$ in the first quarter of 2009. The global financial crisis caused Bursa Malaysia to tumble below 1000 percentage points (Abidin and Rasiah, 2009; Gokay, 2009). As a result, the Malaysian currency exchange rate dropped to $92 \%$ in January 2009.

The purpose of this study is to validate the impact before (2007) and beginning (2008) of the crisis on Bursa Malaysia stocks market companies. The stock prices were downloaded from Data stream maintained by Thomson Reuters. There are 850 companies in Bursa Malaysia, but only 77 companies with more than RM500 million market prices are chosen in this study. The data is retrieved for both quarter one of 2007 and 2008 from January 1 to March 31 on a daily trading basis and were used in testing the differences of the covariance structures. The data involved high dimensional data set due to the number of dimension, $p$ (77 companies) is larger than the number of observation $n$ (60 trading days stock prices of quarter one).

\section{Testing the Difference between Two Covariance Structure}

There are many methods available for testing the different between two covariance structure, such as, Box's M statistic (Box, 1949), Jennrich statistic (Jennrich, 1970) and generalized variance statistic (Wilks, 1932). However these methods unable to cater for high dimensional data set. Therefore a new test known as $S^{*}$ statistic that been developed for high dimensional data set proposed by Sharif et al. (2016), was used in testing the covariance structure. Let $S_{1, L}$ and $S_{2, L}$ are lower elements of covariance matrix $S_{1}$ and $S_{2}$, respectively, to test the hypothesis $H_{0}: \Sigma_{1}=\Sigma_{2}$ versus $H_{1}: \sum_{1} \neq \sum_{2}$ and using the following formula: 


$$
S^{*}=\left[\operatorname{vec}\left(S_{1, L}\right)-\operatorname{vec}\left(S_{2, L}\right)^{t} S_{p}^{-1}\left[\operatorname{vec}\left(S_{1, L}\right)-\operatorname{vec}\left(S_{2, L}\right)\right]\right.
$$

Where:

$$
S_{p}=M^{t}\left(I_{p^{2}}+K\right)\left(S_{\text {pooled }, L} \otimes S_{\text {pooled }, L}\right) M
$$

With:

$$
M=\left(m_{i j}\right)=\left\{\begin{array}{l}
1 ;(i, j)=\left(C_{2}^{a}+b, b\right) \text { for } b=1,2, \ldots, a \\
0 ; \text { elsewhere }
\end{array}\right.
$$

In general, the duplication matrix $M$ can be presented in matrix form as a block matrix $M=\left(M_{1}\left|M_{2}\right| \ldots M_{p}\right)$ of size $\left(k \times p^{2}\right)$ where $k=\frac{1}{2} p(p+1)$ and this matrix can be partitioned into $p$ blocks. Each $M_{r}, r=1,2, \ldots, p$ is a matrix of the size $(k \times p)$ with its elements $m_{i j}$ are defined in (2).

Let $S_{1}$ and $S_{2}$ be the covariance matrices of 77 companies for the first quarter in 2007 and 2008 respectively defined as follows:

$$
\begin{aligned}
S_{1} & =\left[\begin{array}{cccc}
0.0471 & 0.0317 & \cdots & 0.0183 \\
0.0317 & 0.0363 & \cdots & 0.0167 \\
\vdots & \vdots & \ddots & \vdots \\
0.0183 & 0.0167 & \cdots & 0.0104
\end{array}\right] \\
S_{2} & =\left[\begin{array}{cccc}
0.0419 & 0.0226 & \cdots & 0.0133 \\
0.0226 & 0.0186 & \cdots & 0.0092 \\
\vdots & \vdots & \ddots & \vdots \\
0.0133 & 0.0092 & \cdots & 0.0065
\end{array}\right]
\end{aligned}
$$

Since both matrices are symmetric, the lower and upper elements of contain the same elements. Thus, in computing $S^{*}$ statistic, only the lower elements are considered in order to avoid the redundancy and singularity problem. Based on that two matrices, the following pooled covariance matrix, $S_{\text {pooled }}$ is obtained where $S_{\text {pooled }}=\frac{1}{n}\left(n_{1} S_{1}+n_{2} S_{2}\right)$ :

$$
S_{\text {pooled }}=\left[\begin{array}{cccc}
0.0481 & 0.0341 & \cdots & 0.0268 \\
0.0341 & 0.0404 & \cdots & 0.0334 \\
\vdots & \vdots & \ddots & \vdots \\
0.0268 & 0.0334 & \cdots & 0.0405
\end{array}\right]
$$

Based on the above information, the values of $S^{*}=$ 70.34 are determined. Referring to Chi-square distribution, the value of $\chi_{\alpha ; r}^{2}=31.31$ for $\alpha=0.05$. Therefore, the null hypothesis is rejected which implies that both covariance structures are significantly different which leads to identifying which companies influence the stability of stock exchange using optimal minimum spanning tree, network topology and degree centrality measure.

\section{Optimal Minimum Spanning Tree}

There are a few methods to monitor the structure of interrelated variables. For example, principal component analysis (Loretan, 1997), neural network (Fu and Kara, 2011; Kara et al., 2011), minimum spanning tree (Sharif et al., 2012; Rostrup et al., 2013; Setiawan, 2014) and optimal minimum spanning tree (Djauhari, 2013; Djauhari and Gan, 2015). In this case, every stock is considered as a complex network system consisting of 77 stocks as nodes connected by several numbers of links. Each of links is related to the correlation coefficient between the two nodes adjacent to it. Later, the nodes and links will be considered as a network (Sharif et al., 2012).

Let $X_{i}$ and $\left\langle X_{i}\right\rangle$ denote the $i$-th characteristic and the average of $X_{i}$, respectively; $i=1,2, \ldots, p$. The correlation networks are summarized in correlation matrix $C$. It is a symmetric matrix of size $p \times p$ where the element in $i$-th row and $j$-th column is:

$$
\rho_{i j} \frac{X_{i} X_{j}-X_{i} X_{j}}{\sqrt{\left(X_{i}^{2}-X_{i}^{2}\right)\left(X_{j}^{2}-X_{j}^{2}\right)}}
$$

The correlation coefficient of vectors $X_{i}$ and $X_{j}$ that serves to quantify the degree of their linear relationship. By definition, $p_{i i}=1$ for all $i$ and $p_{i j}$ is between -1 to 1 for all $i \neq j$. Thus, $C$ is a numerical summary representing the complex system of characteristics together.

In general, the standard practices to use this method are as follows:

(i) Transform $C$ into a distance matrix $D$ where

$$
D_{i j}=\sqrt{2\left(1-\rho_{i j}\right)} \text { for all } i, j=1,2, \ldots, n
$$

(ii) Find an optimal MST in $D$ which represents the filtered important information contained in $D$

(iii) Analyse the topological property of all characteristics using degree centrality (Borgatti, 1995)

Let $A$ be the adjacency matrix with element $a_{i j}(u)=1$ if the nodes are links in the MST from node $i$ to node $j$, otherwise $a_{i j}(u)=0$. Therefore, the degree centrality of node $u$ is denoted by:

$E_{(u)}=\sum a_{i j}(u)$ 
(iv) Solve (i)-(iii) using Matlab and simplify complex structure using Pajek software

\section{OMST Algorithm}

From fuzzy relation view point, these properties show that $D$ is a symmetric and antiflexible fuzzy relation with $D$ as the membership function. Subsequently, the SDU of $D$ is the min-max transitive closure denoted by $D^{* K}$ for an integer; $2 \leq K \leq n$ (Djauhari and Gan, 2015).

By using $D^{*}$, we construct the forest of all MSTs in $D$. Let $F$ be a forest of all MST and $\left(i=i_{1}, i_{2}, \ldots \ldots, i_{p}=j\right)$ be the chain from $i$ to $j$ in $F$. If we define the distance $d$ between $i$ and $j$ in $F$ by:

$$
\hat{d}(i, j) \underset{\substack{v \\ k=1}}{p-1} d\left(i_{k} i_{k+1}\right)
$$

Then, $D$ is the SDU of $D$. Therefore, there exists an integer $f ; 1 \leq f<p$; such that $d^{* k}(i, j)=\hat{d}(i, j)=d\left(i_{f}, i_{f+1}\right)$.

Let $\Delta$ be a fuzzy relation where its membership function is given by:

$$
\delta(i, j)=\left\{\begin{array}{l}
1 ; d(i, j)-d^{* K}(i, j)=0 \text { and } i \neq j \\
1 ; d(i, j)-d^{* K}(i, j)=0 \text { and } i \neq j
\end{array}\right.
$$

Then, $\Delta$ is the adjacency matrix that corresponds to the forest of all MSTs in $D$. In other words, that forest is defined by all pairs $(i, j)$ where $i>j$ and $\delta(i, j)=1$. Consequently, forest of all MST in $D$ is unique if and only if the number of pairs $(i, j)$, where $i>j$ and $\delta(i, j)=1$, is $n-1$. Based on (7), we find the optimal minimum spanning tree (Djauhari and Lee, 2012; Djauhari, 2013).

\section{Findings and Discussion}

\section{OMST Results}

This section presents the comparison network between the first quarter of 2007 and the first quarter of 2008 in identifying which companies are causing the differences of the covariance structure. In this analysis the information of $77 \times(77-1) / 2=2926$ correlation coefficients is considered to be filtered by using OMST algorithm.

There are 90 and 87 number of links were produced in 2007 and 2008, respectively. To elaborate the findings more clearly, we present the centrality measure for both network. In Fig. 1 and 2, companies are differentiated by different colour and size subject to their centrality scores as presented in Table 1. The centrality scores are arranged from largest to smallest scores.

In the first quarter of 2007 as in Fig. 1 and Table 1, HWAN (green) has the highest number of links in the network with 11 scores which indicates as the most dominant company. It was followed by the second highest HONG (white) and IGOB (white) with 6 scores respectively. This means that in 2007 the rise or fall (instability) of HWAN, HONG and IGOB will give large impact on the stability structure of the stock market. Since there are interrelated to each other, the companies around of these dominant companies will be directly influenced once the occurrence of global financial crisis. The company that only has one (1) score was less significant to the network and more likely to be influenced by the other companies.

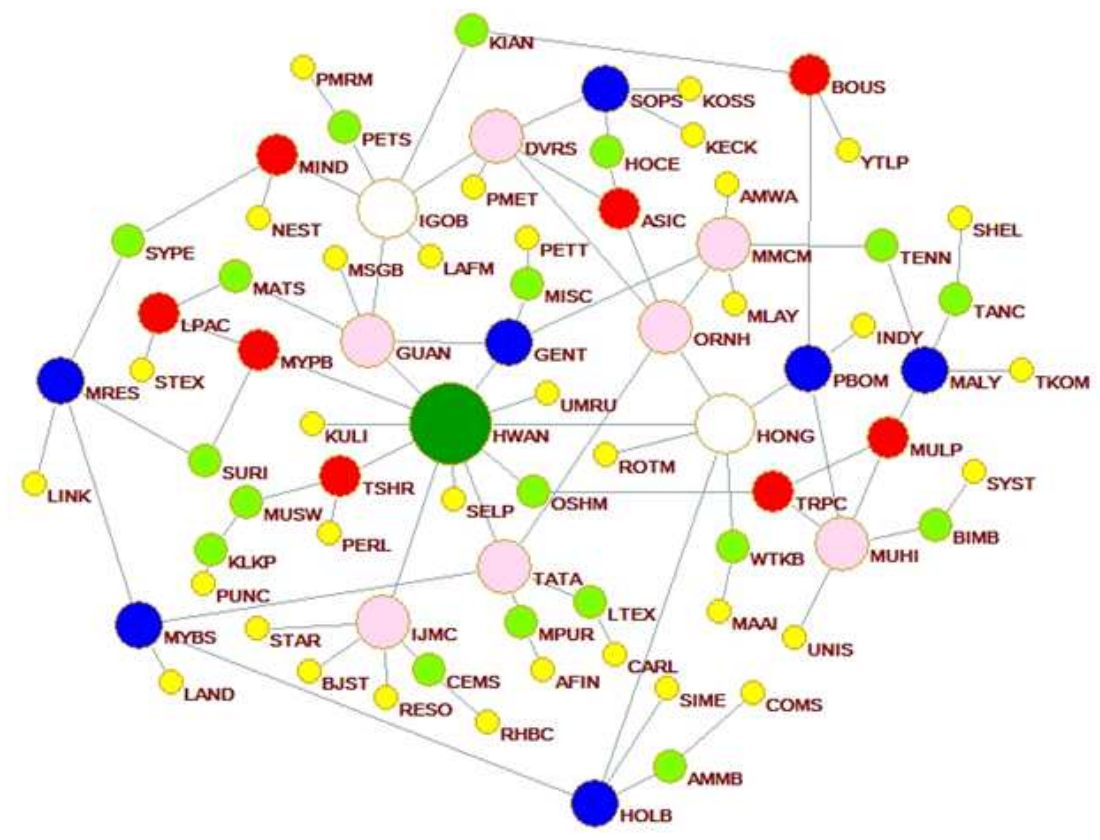

Fig. 1. OMST first quarter of 2007 


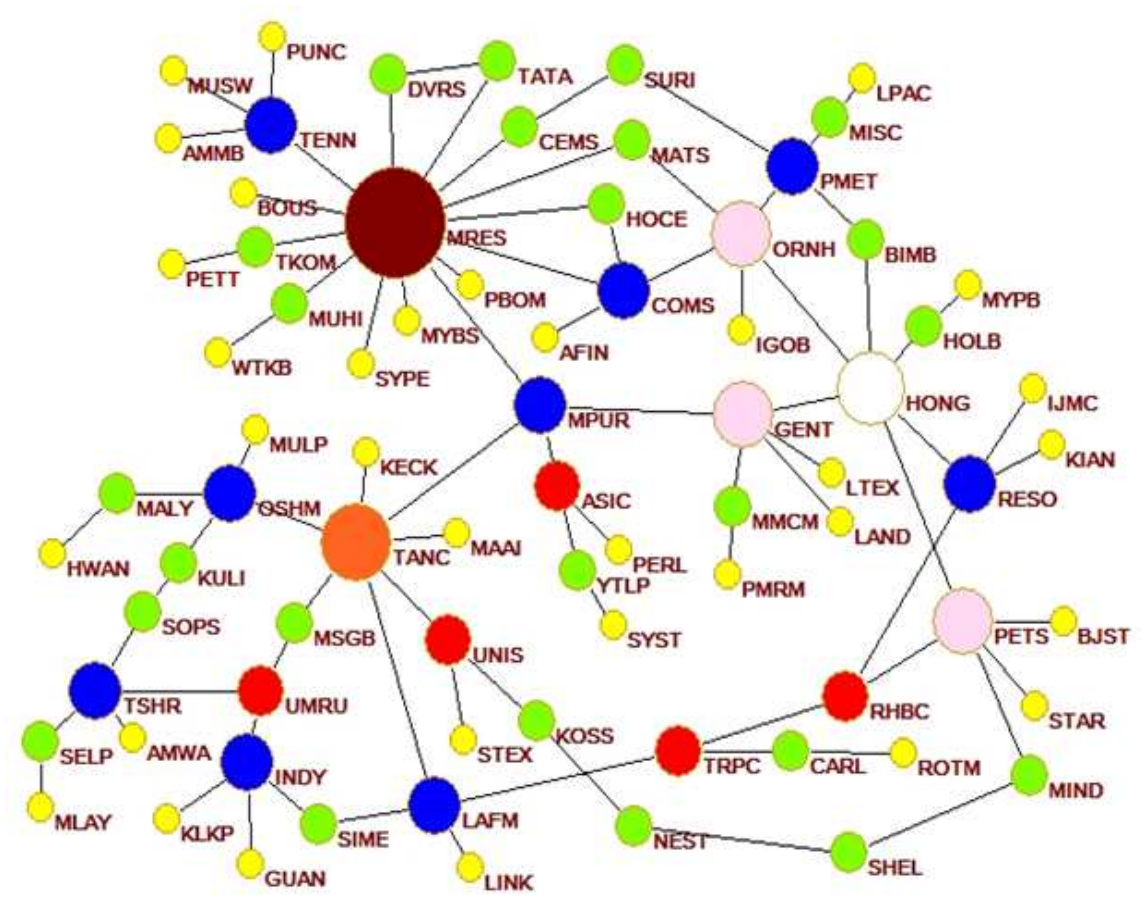

Fig. 2. OMST first quarter of 2008

Table 1. Degree centrality of bursa Malaysia stock price of 2007 and 2008

\begin{tabular}{|c|c|c|c|c|c|c|c|c|c|c|c|}
\hline Code & 2007 & 2008 & Code & 2007 & 2008 & Code & 2007 & 2008 & Code & 2007 & 2008 \\
\hline AFIN & 1 & 1 & HONG & 6 & 6 & MIND & 3 & 2 & SOPS & 4 & 2 \\
\hline MYPB & 3 & 1 & HWAN & 11 & 1 & MRES & 4 & 14 & STEX & 1 & 1 \\
\hline AMMB & 2 & 1 & IGOB & 6 & 1 & MISC & 2 & 2 & SYST & 1 & 1 \\
\hline AMWA & 1 & 1 & IJMC & 5 & 1 & MMCM & 5 & 2 & SELP & 1 & 2 \\
\hline BJST & 1 & 1 & INDY & 1 & 4 & MUHI & 5 & 2 & SHEL & 1 & 2 \\
\hline BIMB & 2 & 2 & KECK & 1 & 1 & MULP & 3 & 1 & SIME & 1 & 2 \\
\hline BOUS & 3 & 1 & KIAN & 2 & 1 & NEST & 1 & 2 & SYPE & 2 & 1 \\
\hline ROTM & 1 & 1 & KOSS & 1 & 2 & ORNH & 5 & 5 & STAR & 1 & 1 \\
\hline CEMS & 2 & 2 & KLKP & 2 & 1 & OSHM & 2 & 4 & SURI & 2 & 2 \\
\hline CARL & 1 & 2 & KULI & 1 & 2 & MATS & 2 & 2 & TATA & 5 & 2 \\
\hline COMS & 1 & 4 & LAFM & 1 & 4 & PMRM & 1 & 1 & TANC & 2 & 7 \\
\hline MUSW & 2 & 1 & LAND & 1 & 1 & PETS & 2 & 5 & TKOM & 1 & 2 \\
\hline DVRS & 5 & 2 & LINK & 1 & 1 & PETT & 1 & 1 & TENN & 2 & 4 \\
\hline MLAY & 1 & 1 & LPAC & 3 & 1 & LTEX & 2 & 1 & TRPC & 3 & 3 \\
\hline GENT & 4 & 5 & MPUR & 2 & 4 & PERL & 1 & 1 & TSHR & 3 & 4 \\
\hline RESO & 1 & 4 & MSGB & 1 & 2 & PMET & 1 & 4 & UNIS & 1 & 3 \\
\hline ASIC & 3 & 3 & MALY & 4 & 2 & PBOM & 4 & 1 & UMRU & 1 & 3 \\
\hline GUAN & 5 & 1 & MAAI & 1 & 1 & PUNC & 1 & 1 & WTKB & 2 & 1 \\
\hline HOCE & 2 & 2 & MYBS & 4 & 1 & RHBC & 1 & 3 & YTLP & 1 & 2 \\
\hline HOLB & 4 & 2 & & & & & & & & & \\
\hline
\end{tabular}

On the other hand, in the first quarter of 2008 as in Fig. 2, MRES (brown) has the highest scores (14) which indicate as the most dominant company. It was followed by the second highest, TANC (orange, 7 scores) and the third highest HONG (white, 6 scores). This result showed that there was a changed in the structure before (2007) and the beginning (2008) of global financial crisis. It was obvious as displayed by Fig. 1 and 2 where the network topology of the companies was totally different.
Based on Table 1, we computed the difference of scores between those two networks. The highest different in the scores was HWAN and MRES. Both share the same score which was 10 but with different role. HWAN has changed from the most dominant (11) to be the less dominant (1), while MRES has changed from the less dominant (4) to the most dominant (14). The same behaviour was also faced by IGOB and TANC. IGOB shifted 5 scores from 6 scores to 1 scores 
and TANC shifted 5 scores from 2 score to 7 score. As for HONG has a score of 0 . Based on these scores, the global financial crisis in 2008 affected HWAN but HONG seems to maintain in the network.

\section{Concluding Remarks}

HWAN was the most dominant in 2007 before the financial crisis but the scenarios changed in the beginning of the crisis in 2008 where MRES was the most dominant but HONG maintain in both situations. This finding of companies was triggered by the new test known as $S^{*}$ statistic that been developed for high dimensional data set which existed in this study. The structure changed was significantly detected by the new test and lead to identifying which company affected by the global financial crisis. Thus, highlighted the importance of the new covariance test ( $S^{*}$ statistic) for handling high dimensional data set and optimal minimum spanning tree, degree centrality measure and network topology in visualizing and monitoring the difference of Bursa Malaysia structure stocks market.

\section{Acknowledgement}

We wish to express our gratitude to Universiti Utara Malaysia for the financial support under the PBIT Research Grant Scheme and RIMC for facilitating the management of the research.

\section{Author's Contributions}

Shamshuritawati Sharif: Her main contribution is in research design, statistical test and writing the manuscript.

Suzilah Ismail: Her main contributions are research discussion and reviewing the manuscript.

Zurni Omar: His main contributions are mathematical concept and reviewing manuscript.

Low Huey Theng: Her main contributions are data collection and data analysis.

\section{Ethics}

This article is original and contains unpublished material. The corresponding author confirms that all of the other authors have read and approved the manuscript and no ethical issues involved.

\section{References}

Abidin, M.Z. and R. Rasiah, 2009. Introduction. Global Financial Crisis Malaysia Econ. Impact Responses, 7-8: 23.

Borgatti, S.P., 1995. Centrality and AIDS. Connections, 18: $112-114$.
Box, G.E.P., 1949. A general distribution theory for a class of likelihood criteria. Biometrika, 36: 317-346. DOI: $10.1093 /$ biomet/36.3-4.317

Djauhari, M.A. and G.S. Lee, 2013. Network topological property of English dialects similarity: A robust filter approach. Am. J. Applied Sci., 10: 646-653. DOI: 10.3844/ajassp.2013.646.653

Djauhari, M.A. and S.L. Gan, 2015. Optimality problem of network topology in stocks market analysis. Phys. A: Statist. Mechan. Applic., 419: 108-114. DOI: $10.1016 /$ j.physa.2014.09.060

Djauhari, M.A., 2012. A robust filter in stock networks analysis. Phys. A: Statist. Mechan. Applic., 391: 5049-5057. DOI: 10.1016/j.physa.2012.05.060

Dolphin, T. and L. Chappell, 2010. The effect of the global financial crisis on emerging and developing economies.

Ergungor, O.E. and K. Cherny, 2009. Effective practices in crisis resolution and the case of Sweden. Economic Commentary.

Fu, L. and L.B. Kara, 2011. Neural network-based symbol recognition using a few labeled samples. Comput. Graph., 35: 955-966. DOI: 10.1016/j.cag.2011.07.001

Gokay, B., 2009. Tectonic Shifts and Systemic Faultlines: A Global Perspective to Understand the 2008-2009 World Economic Crisis. Alternatives: Turkish J. Int. Relations, 8: 19-58.

Jennrich, R.I., 1970. An asymptotic $\chi 2$ test for the equality of two correlation matrices. J. Am. Statist. Associat., 65: 904-912. DOI: 10.2307/2284596

Kara, Y., M. Acar Boyacioglu and Ö.K. Baykan, 2011. Predicting direction of stock price index movement using artificial neural networks and support vector machines: The sample of the istanbul stock exchange. Expert Syst. Applic., 38: 5311-5319. DOI: $10.1016 /$ j.eswa.2010.10.027

Loretan, M., 1997. Generating market risk scenarios using principal components analysis: Methodological and practical considerations. Federal Reserve Board.

Rostrup, S., S. Srivastava and K. Singhal, 2013. Fast and memory-efficient minimum spanning tree on the GPU. Int. J. Comput. Sci. Eng., 8: 21-33.

DOI: $10.1504 /$ IJCSE.2013.052115

Setiawan, K., 2014. Global stock market landscape: An application of minimum spanning tree technique. Int. J. Operat. Res., 20: 41-67.

Sharif, S., N.S. Yusoff and M.A. Djauhari, 2012. Network topology of foreign exchange rate. Modern Applied Sci., 6: 35-35. DOI: 10.5539/mas.v6n11p35

Sharif, S., S. Ismail and Z. Omar, 2016. Automated control chart for high dimension data set. Universiti Utara Malaysia, Technical Report.

Wilks, S.S., 1932. Certain generalizations in the analysis of variance. Biometrika, 24: 471-494.

DOI: $10.1093 /$ biomet/24.3-4.471 